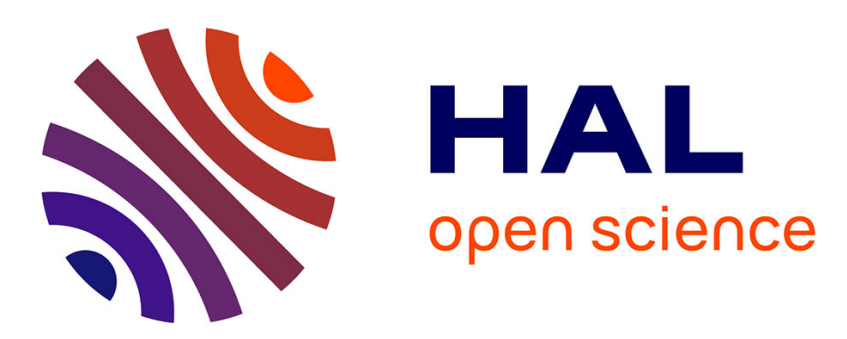

\title{
Journalisme et fiction dans le roman argentin, de Miguel Bonasso à Tomás Eloy Martinez
}

\author{
Isabelle Bleton-Bonnet
}

\section{To cite this version:}

Isabelle Bleton-Bonnet. Journalisme et fiction dans le roman argentin, de Miguel Bonasso à Tomás Eloy Martinez. América: cahiers du CRICCAL, 2000, 24 (1), pp.139-148. 10.3406/ameri.2000.1460 . halshs-02135424

\section{HAL Id: halshs-02135424 \\ https://shs.hal.science/halshs-02135424}

Submitted on 21 May 2019

HAL is a multi-disciplinary open access archive for the deposit and dissemination of scientific research documents, whether they are published or not. The documents may come from teaching and research institutions in France or abroad, or from public or private research centers.
L'archive ouverte pluridisciplinaire HAL, est destinée au dépôt et à la diffusion de documents scientifiques de niveau recherche, publiés ou non, émanant des établissements d'enseignement et de recherche français ou étrangers, des laboratoires publics ou privés. 


\section{Journalisme et fiction dans le roman argentin, de Miguel Bonasso à}

\section{Tomás Eloy Martinez}

\section{Isabelle Bleton}

\section{Resumen}

«Periodismo y ficción en la novela argentina : de Miguel Bonasso a Tomás Eloy Martinez ».

En estos tiempos en que la manifestación de la violencia politica en Argentina y en el mundo trastornan profundamente el modo de aprehender la realidad, asi como la confianza que se ténia en el realismo tradicional, van surgiendo en Argentina unas nuevas formas literarias que intentan plasmar la historia reciente del pais. Ambos periodistas, Miguel Bonasso, Recuerdo de la muerte (1984) y Tomás Eloy Martinez, La novela de Perón (1985) y Santa Evita (1995), tratan de poner al servicio de una pesquisa sobre el pasado las técnicas periodisticas tanto como las novelisticas, integrando fiction y relato documental, en una transgresión espectacular de las ultimas fronteras que separaban el relato de lo referente. Hacen coexistir unos pactos de lectura contradictories y los personajes históricos con los personajes de ficción. Esta escritura cobra, ademâs, una dimension autorreflexiva : la escritura es un componente de la historia narrada. El acto mismo de representar la realidad se vuelve tema, fin problemático de la escritura y de la lectura.

\section{Citer ce document / Cite this document :}

Bleton Isabelle. Journalisme et fiction dans le roman argentin, de Miguel Bonasso à Tomás Eloy Martinez. In: América : Cahiers du CRICCAL, n²4, 2000. Les nouveaux réalismes, v1. pp. 139-148;

doi : https://doi.org/10.3406/ameri.2000.1460

https://www.persee.fr/doc/ameri_0982-9237_2000_num_24_1_1460

Fichier pdf généré le 16/04/2018 


\section{Journalisme et fiction dans le roman argentin, de Miguel Bonasso à Tomás Eloy Martínez}

$\mathrm{D}$

ans la production romanesque argentine postérieure au régime dictatorial du Proceso de Reorganización Nacional' se distinguent des textes dont l'objet est la reconstitution méticuleuse de faits du passé récent, et qui se situent à la frontière entre le roman et le texte journalistique. Bien que le référent traité soit réel, des procédés de fictionalisation rendent possible une lecture romanesque. Recuerdo de la muerte de Miguel Bonasso (publié en 1984), La novela de Perón (1985) et Santa Evita (1995) ${ }^{2}$ de Tomás Eloy Martínez, sont des romans où semble émerger une forme nouvelle de représentation du réel, à mi-chemin entre texte de fiction et document. Une double filiation peut être identifiée. D'une part, un mouvement de rénovation du journalisme, qui s'est développé dès la fin des années 60 aux Etats-Unis, appelé le «New Journalism », et d'où est issue une forme littéraire, la «non fiction novel », dont l'exemple emblématique est De sang-froid, de Truman Capote (1966), et d'autre part, l'œuvre de journalisme d'enquête de Rodolfo Walsh, constituée par la trilogie des récits d'enquête: Operación masacre (1957), El caso Satanowsky (1958) et ¿Quién mató a Rosendo? (1969). Avec ses témoignages politico-littéraires ${ }^{3}$, Walsh a été considéré comme le fondateur de la « novela de no-ficción » latino-américaine 4 . Cette filiation est présente dans nos textes. Bonasso le mentionne comme mi maestro Rodolfo $\mathrm{Walsh}^{5}$ et fait référence à sa célèbre «Carta abierta de un escritor a la Junta Militar ${ }^{6}$, écrite en 1977 , peu avant sa disparition. Tomás Eloy Martínez non seulement l'intègre comme personnage dans Santa Evita, mais établit une filiation marquée entre ce roman et le conte de Walsh «Esa mujer $»^{7}$, texte qui fait implicitement allusion au cadavre embaumé d'Eva Perón. Santa Evita peut être lu comme le développement du conte de Walsh, comme l'aboutissement de l'enquête

1. Ce régime, mis en place en 1976 par une junte de généraux, mettait fin à la présidence d'Isabel Perón, qui avait assumé le pouvoir à la mort du géneral Perón en 1975. Le Proceso dura jusqu'en 1983, lorsque la défaite dans la Guerre des Malouines acheva de discréditer le régime. Un régime démocratique, sous la présidence de Raúl Alfonsín, lui succéda.

2. Nous faisons référence ici aux éditions suivantes : Recuerdo de la muerte Buenos Aires, Planeta, 1994 ; La novela de Perón, Buenos Aires, Planeta, 1991; Santa Evita, Buenos Aires, Planeta, 1995.

3. L'expression est de Pedro Orgambide : "La narrativa de Walsh », Plural, N 117/10-9, México, 1981.

4. José Emilio Pacheco, «Rodolfo Walsh desde México », prólogo a Obra literaria completa, México, Siglo XXI, 1981.

5. Miguel Bonasso, Recuerdo de la muerte, p. 444.

6. Ibid., p. 52 et 57 . Rodolfo Walsh, "Carta abierta de un escritor a la Junta Militar », in Roberto Baschetti, Rodolfo Walsh, vivo, Buenos Aires, Ed. de la Flor, 1994, p. 241-253.

7. Rodolfo Walsh, Obra literaria completa, op. cit. 
amorcée par le personnage de ce conte. La figure de Walsh incarne ainsi non seulement le modèle du journaliste enquêteur engagé dans un compromis total avec la vérité, mais aussi un père littéraire.

Recuerdo de la muerte de Miguel Bonasso, publié en 1984 à Buenos Aires, a pour thème la répression militaire menée dans un des camps de concentration les plus célèbres, celui de l'Ecole de Mécanique de l'Armée (communément désignée par le sigle ESMA), pendant la dictature du Proceso. Le récit se base sur l'histoire réelle d'un détenu, Jaime Dri. Le texte paraît alors que le pays vient de retrouver un régime démocratique et que la société commence à découvrir des témoignages sur les crimes du terrorisme d'Etat. La novela de Perón de Tomás Eloy Martínez, paru en 1985, traite non pas du Proceso mais de la période antérieure, notamment du massacre de l'aéroport d'Ezeiza, lors du retour du Général Perón en Argentine, le 20 juin 1973. Mais il traite aussi de la vie de Perón: en particulier son enfance, son adolescence, sa carrière militaire jusqu'à son accession à la présidence en 1945. Le roman reconstitue la face cachée du Général Perón, opérant à travers de nombreux témoignages et documents une démythification ou démystification du personnage, et proposant une réflexion historique sur l'échec du péronisme. En 1995, Tomás Eloy Martínez consacre un roman à Eva Perón cette fois : Santa Evita. L'auteur reconstitue l'histoire hallucinante du cadavre embaumé d'Evita, qui avait été séquestré par l'armée en 1955, puis caché, pour n'être rendu à Perón qu'en 1971. En alternance, sont reconstitués, sur la base de témoignages, des fragments méconnus de la biographie d'Eva Perón. Apparaît aussi un troisième espace narratif : le récit de l'enquête dont le héros est l'auteur luimême.

Ces trois textes ont en commun le fait de mêler étroitement deux types de démarche. D'abord, une forme de réalisme issue de la pratique du journalisme : représentation de faits réels, entretiens avec des témoins, montage de documents, procédés conduisant à la postulation d'un pacte de lecture référentiel ou documentaire. Ensuite, un discours de fiction, issu de la pratique littéraire, sous forme d'une recréation fictionnelle d'événements tantôt attestés par des témoignages, tantôt inventés librement. Cette écriture postule alors également un pacte de lecture romanesque : le lecteur n'est pas obligé de faire le lien avec la réalité, il peut choisir de lire le livre " comme un roman ". Il existe donc une double possibilité de décodage, un double pacte de lecture.

Bonasso, dans l'un des deux épilogues de Recuerdo de la muerte, définit son roman comme «novela-real» ou « realidad-novelada ${ }^{\mathbf{8}}$, et le paratexte propose l'expression "novela testimonial de tema criminal». L'auteur justifie le recours à la forme romanesque pour décrire un référent réel de la façon suivante : 
No es por azar, tampoco, que [el texto] asumió la forma novelística. La narración muestra, no demuestra. La novela permite desenterrar ciertos arcanos que a veces se niegan a salir dentro de las pautas más racionales de la crónica histórica, el testimonio de denuncia o el documento político. Pero la voluntad de novelar no encubre aquí el designio de modificar los hechos. Todo lo que se dice es rigurosamente cierto y está apoyado sobre una base documental enorme y concluyente. ${ }^{9}$

Le terme de «novela » est revendiqué, sans être conçu comme contradictoire avec une lecture référentielle. Le roman est perçu comme un espace narratif suffisamment ouvert et souple pour offrir des possibilités que n'offrent pas des forme non fictionnelles, en particulier le pouvoir de « desenterrar arcanos » déterrer les arcanes des faits, du vécu. Le point de vue de Tomás Eloy Martínez est très proche. Dans le titre La novela de Perón, le mot «novela » implique « ne lisez pas cela comme de l'histoire », alors que le mot «Perón », par son poids référentiel, nous tire vers un tout autre horizon d'attentes : la biographie, l'histoire. En outre, une citation tirée d'un texte d'Hemingway livre une clef de lecture :

Si el lector lo prefiere, puede considerar este libro como una obra de ficción. Siempre cabe la posibilidad de que un libro de ficción deje caer alguna luz sobre las cosas que antes fueron narradas como hechos.

Ernest HEMINGWAY, prefacio de Paris era una fiesta

On nous propose donc ne pas opposer fiction et vérité mais de les concevoir comme un système complémentaire, et comme une sorte de défi lancé au lecteur. Cette sollicitation du lecteur est une caractéristique des textes. Dans un entretien accordé à la revue Crisis en 1988, Martínez argumente $\mathrm{d}$ 'une manière comparable à celle de Bonasso :

Lo que yo descubri es que si buscás la verdad de un personaje a través de conjeturas que se desprenden de las mismas huellas que deja, te podés acercar a su verdad central más certeramente que a partir de documentos. En los documentos, en los restos de historia que cada uno deja de sí, hay zonas que deliberadamente quedan a oscuras; verdades profundas que si te acercás narrativamente a través de una serie de conjeturas, podés ver de un modo inesperado. ${ }^{10}$

Cette déclaration explique la démarche de La novela de Perón: lorsque les documents laissent des zones d'ombre, la narration fictionnelle s'empare alors de ces vides et permet d'atteindre des vérités profondes, des « arcanes », comme dit Bonasso. C'est ainsi que Martínez a approché le personnage de Perón.

Dans Santa Evita, la condition de roman est d'emblée affirmée par le sous-titre de la couverture : «novela ». Dans un entretien publié dans $E l$ Cronista Cultural en août 1995, Tomás Eloy Martínez établit la différence entre La novela de Perón et Santa Evita. Alors que dans La novela de Perón,

9. Ibid., p. 443.

10. Tomás Eloy Martínez, « El poder escribe la historia », Crisis, 62, 1988, p. 35. 
on avait une enquête journalistique retravaillée par les ressources du roman, Santa Evita est construit selon la démarche inverse :

Aquí, lo que sucede es a la inversa. Hay una novela trabajada con algunos métodos del periodismo, de la cultura popular, del cine, de la sociología y de la historia. Y si bien los hechos básicos pertenecen al acervo histórico - el renunciamiento de Eva Perón, el itinerario de su cuerpo - la construcción del texto es a la vez la reconstrucción del mito. Lo que a mí me importaba es dejar escrito todo lo que ese cuerpo ha ido sembrando en la imaginación, en la memoria, en los deseos de los argentinos. ${ }^{11}$

Il s'agit cette fois non pas de démythifier, comme dans La novela de Perón, et de rétablir des vérités historiques, mais bien de se plonger dans le mythe même d'Evita. Cependant, le lecteur trouve tous les éléments pour établir un pacte de lecture référentiel : mentions de sources, de références bibliographiques. Par les interventions récurrentes du narrateur-enquêteur, par tout un appareil de notes et de commentaires, le lecteur est constamment incité à aller vérifier la véracité des faits racontés. L'effet de réalité est accentué par l'identification explicite entre l'auteur et le narrateur. Mais plutôt qu'une dimension autobiographique, cette constante autodénomination mène à une forte autoréférentialité du texte.

Comme on vient de le voir, cette double possibilité de lecture, référentielle et romanesque, proposée par nos trois textes, repose sur la création d'un espace référentiel au sein du roman, ou inversement, d'un espace romanesque entre les documents. Tous les textes ont une double référence : externe et interne. La référence externe est constituée par la corrélation entre texte et documents réels, texte et savoir historique du lecteur, et la référence interne par la manière dont les faits sont représentés et créent des résonnances fictionnelles, convoquant chez le lecteur des références littéraires. Cette double référentialité, et la tension qui en résulte, serait une caractéristique de ce nouveau réalisme proposé par les textes.

Cette double référence n'est pas sans soulever des problèmes. Les auteurs justifient l'invention de scènes en invoquant la fiction comme mode de connaissance qui va au-delà des apparences, qui permet de sonder des profondeurs. Mais comment s'articulent les différents discours ? Comment se configure un discours romanesque qui doit mener à une vérité sur le référent ?

Avant tout, leurs stratégies narratives sont conditionnées par la nature même du référent choisi. Le discours de l'enquête dans les textes de Walsh se définissait par rapport à une version préalable des faits, une version insatisfaisante, pour différentes raisons, qui entraîne un besoin de rectification, de correction. Selon Ana María Amar Sánchez, les récits d'enquête de Walsh

11. Osvaldo Quiroga, entretien avec Tomás Eloy Martínez, El Cronista cultural, 4/8/1995, p. 3. 
[...] son relatos de investigaciones acerca de hechos que, aunque reales y quizá por eso, son percibidos casi como inverosímiles: el lector se ve obligado a confrontarlos constantemente con su experiencia histórica y literaria ${ }^{12}$.

Walsh se propose de traiter lo omitido, censurado o deformado por la prensa $^{13}$. Le référent traité est moins constitué par des faits que par les versions qui circulent de ces faits. On retrouve le même statut du référent chez Bonasso et Martínez. Le référent de Recuerdo de la muerte, basé en grande partie sur des témoignages, souffre d'un manque de reconnaissance officielle, d'une marginalisation. Tout ce que révèle Bonasso sur la vie dans les prisons secrètes de l'armée est trop stupéfiant. Dans le cas des textes de Tomás Eloy Martínez, le référent présente aussi cette nature incertaine, cette fois non pas à cause d'un déficit d'information, mais à cause d'une prolifération de récits, rumeurs, versions, légendes, qui rendent extrêmement difficile l'accès à la vérité.

Dans tous les cas, le référent est de nature éminemment discursive. C'est déjà une élaboration du discours historique, du discours social, ainsi que du discours du pouvoir. La problématique du référent se pose en terme d'intertextualité, de rapport du texte avec d'autres textes.

Comment les auteurs vont-ils résoudre le problème de l'invraisemblance du référent ? Comment faire accéder ce référent douteux, voire clandestin, au statut de fait établi? Bonasso va tenter de résoudre le problème de l'invraisemblance du référent par l'adoption d'une forme de narration réaliste. Dans Recuerdo de la muerte, il narrativise les faits, a recours à tout l'éventail des procédés du roman réaliste, à la manière de $D e$ sang-froid. Sur le plan de la forme, il sépare nettement dialogue et récit, adopte une narration linéaire, structure clairement la composition de son roman. Sur le plan de la fiction, il construit ses personnages selon la vraisemblance psychologique. Au lieu d'insister, comme le fait Martínez, sur le montage de documents provenant de la réalité, Bonasso privilégie la narrativisation de l'avant-texte. Il reprend les informations données par les documents et les témoignages et les intègre dans un récit au passé, de type réaliste. Son écriture fusionne les informations réelles et la fiction vraisemblable en un récit uniforme. Mais alors qu'un roman réaliste ne prétend pas à une valeur référentielle, mais seulement à un effet de réel, dans le texte de Bonasso il s'agit d'authentifier le récit afin qu'il soit décodé comme un texte référentiel et non comme un texte de fiction qui veut imiter le vrai. Le vraisemblable doit déboucher sur l'authentique, le factuel. On lit finalement le réel comme une fiction, en ayant pleinement conscience de ce paradoxe. Cette lecture "en connaissance de cause » distingue un texte

12. Ana Maria Amar Sánchez, "La propuesta de una escritura (en homenaje a Rodolfo Walsh)» in Revista iberoamericana, 135-136, 1986, p. 435.

13. Ana María Amar Sánchez,El relato de los hechos, Rodolfo Walsh: Testimonio y escritura, Rosario, Beatriz Viterbo Editora, 1992, p. 55. 
comme Recuerdo de la muerte d'un récit réaliste de faits imaginaires, et constitue un trait définitoire du genre « novela de no-ficción » ${ }^{14}$.

Martínez, au lieu de fusionner le réel dans un récit homogène, propose la méthode du duelo de versiones narrativas. Le roman reprend des versions officielles de l'histoire et leur oppose d'autres versions issues de témoignages privés. Dans leurs intervalles, la fiction construit une cohérence. C'est ce qu'il appelle, dans ce même article de Crisis, «la apuesta de la literatura »:

La historia brinda siempre la versión oficial de los hechos. El poder escribe la historia. La literatura busca abrirse una fisura, trata de engendrar un discurso, a partir de la historia, en el cual se dé una versión que se superponga a la de la historia oficial y triunfe. Esa es la apuesta de la literatura. A mí ese duelo entre versiones narrativas entre la ficción y la historia me pareció particularmente rico cuando Perón me dicta su autobiografia por un hecho totalmente azaroso ${ }^{15}$.

Ici Martínez fait allusion à son propre travail journalistique : en 1970 il a réalisé de longs entretiens avec le général Perón, qui résidait alors à Madrid. Ce texte autobiographique fut publié dans la revue Panorama. Or, c'est ce texte qui est repris dans La novela de Perón pour constituer le discours des « Memorias ». Mais ces mémoires occultaient certains faits car elles étaient construites pour l'histoire officielle, pour donner une image édifiante du général, pour servir d'évangile du péronisme. Ces faits, Martínez décide de les reconstituer. A ces «Memorias » édifiantes, il oppose les «contramemorias», sorte de biographie irrespectueuse basée sur des témoignages privés. Et pour dénoncer le caratère falsificateur des "Memorias ", il en attribue l'écriture non pas à Perón, mais à son secrétaire privé, le tristement célèbre López Rega, dit el Brujo, le fondateur de la Triple A. Martínez ne cherche pas à rendre crédible une version, mais à mettre en cause la crédibilité des versions existantes, par le duel de versions. Invité à jongler avec les différents discours, le lecteur est sollicité activement pour participer à la réorganisation du champ référentiel. Dans Santa Evita, parmi la prolifération de vérités possibles, l'auteur choisit de montrer les plus marginales, les plus méconnues, les plus controversées. Ainsi, à la différence du roman réaliste traditionnel, qui invente et s'emploie à rendre vraisemblable son référent fictif, les romans de Martínez partent d'une réalité problématique et insistent sur sa condition invraisemblable. Le roman adopte alors la structure de l'enquête. Dans Santa Evita, la narration réaliste du début s'efface progressivement, infiltrée par les marques de plus en plus

14. Ici, observons que le mot fiction peut prendre deux sens bien différents. Dans l'expression « novela de no-ficción ", "ficción " est synonyme de discours renvoyant à un référent imaginaire, tandis que la "fiction" dont relève l'écriture de Walsh et de Bonasso, est un effet de la narration, une construction faite d'éléments référentiels organisés dans un récit qui diffère peu de celui d'un roman. C'est pourquoi l'expression " novela de no-ficción » est toujours discutée par les critiques, qui lui préfèrent " novela testimonial " ou " novela documental ». Nous penchons plus pour l'expression " roman d'enquête " car plus qu'un document, ce que les textes donnent à lire est une recherche de vérité, dans une structure d'investigation.

15. Tomás Eloy Martínez, « El poder escribe la historia », op. cit., p. 35 
fréquentes de l'enquête: le discours devient conscient d'être la reconstitution des faits. Alors que Bonasso cherchait à dissimuler ces marques de l'enquête, à les fondre dans une narration réaliste, Martínez veut au contraire les montrer ostensiblement et exhiber, mettre au premier plan, l'enquête et les témoignages. Cette démarche rend impossible tout réalisme au sens traditionnel du terme, car le discours ainsi construit est pleinement conscient de sa condition de discours, de construction verbale. Le lecteur est amené à adopter une lecture critique. La novela de Perón et Santa Evita postulent un lecteur modèle symétrique au narrateur-enquêteur : un lecteurenquêteur qui participe, lui aussi, de cette recherche consciente d'une vérité insaisissable.

Ce nouveau réalisme qui se dessine à travers les trois textes s'accompagne d'une dimension autorréflexive et métafictionnelle. Dans Recuerdo de la muerte, cette réflexion apparaît dans un chapitre particulier, où de façon exceptionnelle, le narrateur intervient à la première personne, sous forme d'une confession au lecteur :

Confesión

Perdón por meterme. No puedo evitarlo. Es, tal vez, una falta de pudor. Pero siento que resulta imprescindible. Este capítulo es uno de los que me han dado más trabajo. Y quiero contarle al lector cómo está naciendo. En un anochecer de julio de 1983, en la ciudad de México ${ }^{16}$.

Alternant des séquences historiques et des séquences commentatives dans lesquelles le sujet de l'énonciation exprime son insatisfaction, le chapitre offre un récit de l'écriture même, des difficultés d'écrire le roman. A travers ce dédoublement de l'écriture s'établit un débat, une relation dialectique entre texte narratif et texte critique, entre la figure du narrateurhistorien et celle du critique. Comme le montre Mark Currie dans son ouvrage Metafiction, cette relation dialectique est paradigmatique de la métafiction conçue comme une critique simultanée à la création ${ }^{17}$. En exprimant sa méfiance envers les possibilités mimétiques du langage, le roman de Bonasso s'inscrit dans le cadre de la crise de la représentation réaliste, ou crise du contrat mimétique ${ }^{18}$. La réflexion sur la littérature, présente au sein d'un récit véridique, confirme que toute écriture des faits, si réels soient-ils, ne peut se concevoir sans des stratégies littéraires, sans une position littéraire préalable.

Cette irruption du sujet-narrateur se produit également dans La novela de Perón, dans un chapitre qui condense des commentaires métafictionnels. Le narrateur dit au lecteur son projet d'écriture : "Quiero contar lo no

16. Miguel Bonasso, Recuerdo de la muerte, p. 396.

17. Mark Currie, Metafiction, Longman Lundon \& New York, 1995, p. 3.

18. Voir Ana Maria Barrenechea, "La crisis del contrato mimético en los textos contemporáneos", in Cedomil Goic, Historia y crítica de la literatura hispanoamericana, vol III, Barcelona, Editorial Crítica, 1988, p. 63. 
escrito, limpiarme de lo no contado, desarmarme de la historia para poder armarme al fin con la verdad $»^{19}$. Méfiant envers l'histoire, il affirme le pouvoir cognitif du roman, sa capacité à éclairer la réalité à travers une représentation capable de rivaliser avec l'histoire dans l'accès à la vérité.

Santa Evita est, des trois textes, celui où cette dimension métafictionnelle est la plus développée. Le narrateur, en permanence visible dans le texte, exprime fréquemment sa difficulté à narrer. Il raconte même au lecteur une version antérieure du roman qui a échoué, una larga y descartada versión de esta misma novela :

Nada de eso tenía sentido y casi nada sobrevivió en las versiones que siguieron. Ciertas frases, en las que trabajé durante semanas, se evaporaron bajo el sol de la primera lectura, sajadas por la impiedad de un relato que no las necesitaba ${ }^{20}$.

Il évoque aussi les causes qui ont déterminé la structure complexe du roman, sa narration fluctuante, multiforme, sa temporalité bouleversée, esquissant ainsi une histoire de l'écriture même du roman :

¿Santa Evita iba a ser una novela? No lo sabía y tampoco me importaba. Se me escurrían las tramas, las fijezas de los puntos de vista, las leyes del espacio y de los tiempos ${ }^{21}$.

L'histoire de l'écriture du roman, s'ajoutant à l'histoire du corps et aux fragments biographiques, finit par constituer une sorte d'autofiction. On retrouve là la marque de Borges, qui se fictionnalisait dans certains de ses contes, ainsi que l'écho d'un conte de Ricardo Piglia, "Homenaje a Roberto Arlt ", où l'auteur se représente comme personnage d'une fiction. Martínez ajoute à cela une mise en abyme finale du roman : le narrateur renvoi le lecteur au début en citant la première phrase du livre :

En la soledad de Highland Park, me senté y anoté estas palabras: « Al despertar de un desmayo que duró más de tres días, Evita tuvo al fin la certeza de que iba a morir ${ }^{22}$.

Le texte est circulaire, l'écriture se mord la queue, rappelant une fois de plus la marque de Borges. C'est sur l'autofiction que se termine Santa Evita, et non pas sur l'histoire du cadavre et du colonel nécrophile, ni sur la biographie. Martínez clôt le roman sur sa propre expérience d'écrivainjournaliste en lutte avec l'écriture. Du travail sur les témoignages, les documents, les versions d'un même fait, il ne reste plus que l'acte d'écrire, le geste déterminant, si simple mais si difficile à la fois : 
Desde entonces, he remado con las palabras, llevando a Santa Evita en mi barco, de una playa a la otra del ciego mundo. No sé en qué punto del relato estoy. Creo que en el medio. Sigo, desde hace mucho, en el medio. Ahora, tengo que escribir otra vez ${ }^{23}$.

C'est sur cette annonce d'une écriture à venir que se termine le roman, fin toute provisoire, faux dénouement, qui nous donne l'impression d'un livre en cours d'écriture. Jamais finie, interminable, l'enquête s'avère être une quête : quête de vérité sur les faits, mais avant tout quête de l'écriture, et quête de soi-même.

Les continuateurs du genre inauguré par Rodolfo Walsh ont considérablement fait évoluer ce qui au départ était un récit d'enquête politico-policière. Les auteurs ont poussé jusqu'au bout la subjectivisation, en créant des personnages à part entière, voire en faisant du narrateurenquêteur le protagoniste, comme s'il était le détective privé d'un roman noir sur la réalité. Ils ont donné à des procédés littéraires une légitimité dans l'enquête sur le réel. Bonasso récupère le code du réalisme psychologique et subjectif, le roman comme exploration de l'intériorité, avec toutefois, dans un chapitre à part, une réflexion métafictionnelle qui modère la confiance dans le réalisme. L'écriture de Martínez, elle, est sans cesse traversée par la métafiction, la mise en abyme, la réflexivité, l'intertextualité. La fiction est un questionnement permanent de l'écriture sur elle-même. L'auteur rend visible la géologie de son texte, en mentionnant ses sources, en narrant plusieurs fois une même scène selon des procédés différents, en se représentant dans ses difficultés à écrire le texte que l'on a sous les yeux. La trace de l'écriture borgésienne cohabite avec celle de Walsh, deux écritures pourtant si éloignées.

Bonasso et Martínez ont en commun d'avoir des incertitudes sur le réel, mais au lieu de s'enfermer dans l'antiréalisme, ils tentent de confronter leurs incertitudes avec cette dénommée "réalité ». La représentation du réel passe alors par une réflexion sur la validité même des moyens utilisés. Telle est peut-être l'un des traits dominants de ce nouveau réalisme. D'autre part, ce réalisme semble prendre la forme narrative de l'enquête, en particulier du journalisme d'enquête, avec aussi de nombreuses références à l'enquête policière. Il ne relève plus du réalisme social ou des formes anciennes de réalisme naïf, qui croyaient en l'objectivité. Le " réel », passé ou présent, est dès lors considéré comme un réseau de traces, d'indices, de récits, de légendes. La fiction textualise les incertitudes sur l'expérience, et l'enquête devient le corrélat de la création littéraire. L'enquête compte plus que les résultats, l'exploration compte plus que les explications. Enfin, l'autoconscience de la fiction s'instaure, héritage de la littérature expérimentale, et s'hybride avec le témoignage et le document, héritage du Nouveau 
Journalisme et de Rodolfo Walsh. Il reste aussi le réalisme de la forme même : dans leur forme, les textes sont le miroir des inquiétudes et de la perplexité de l'homme actuel, toujours en quête des sens perdus.

Isabelle BLETON 